\title{
TOWARDS A LOCATION-BASED SERVICE FOR EARLY MENTAL HEALTH INTERVENTIONS IN DISASTER RESPONSE USING MINIMALISTIC TELE- OPERATED ANDROID ROBOTS TECHNOLOGY
}

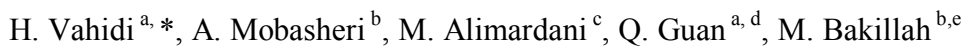 \\ ${ }^{a}$ Faculty of Information Engineering, China University of Geosciences, Wuhan, China - (vahidi, guanf @cug.edu.cn) \\ ${ }^{\mathrm{b}}$ GIScience Research Group, University of Heidelberg, Heidelberg, Germany - \\ (amin.mobasheri, mohamed.bakillah @geog.uni-heidelberg.de) \\ ${ }^{\mathrm{c}}$ Department of System Innovation, Graduate School of Engineering Science, Osaka University, Osaka, Japan - \\ maryam.alimardani@irl.sys.es.osaka-u.ac.jp \\ ${ }^{\mathrm{d}}$ Center for Advanced Land Management Information Technologies, School of Natural Resources, University of Nebraska-Lincoln, \\ Lincoln, USA - qguan2@unl.edu \\ ${ }^{\mathrm{e}}$ Department of Geomatics Engineering, University of Calgary, Alberta, Canada
}

Commission IV

KEY WORDS: Disaster Response, Mental Health, Location-based Service, Web GIS, Tele-operated Android Robots

\begin{abstract}
:
Providing early mental health services during disaster is a great challenge in the disaster response phase. Lack of access to adequate mental-health professionals in the early stages of large-scale disasters dramatically influences the trend of a successful mental health aid. In this paper, a conceptual framework has been suggested for adopting cellphone-type tele-operated android robots in the early stages of disasters for providing the early mental health services for disaster survivors by developing a locationbased and participatory approach. The techniques of enabling GI-services in a Peer-to-Peer (P2P) environment were studied to overcome the limitations of current centralized services. Therefore, the aim of this research study is to add more flexibility and autonomy to GI web services (WMS, WFS, WPS, etc.) and alleviate to some degree the inherent limitations of these centralized systems. A P2P system Architecture is presented for the location-based service using minimalistic tele-operated android robots, and some key techniques of implementing this service using BestPeer were studied for developing this framework.
\end{abstract}

\section{INTRODUCTION}

Providing early mental health services during disaster is a great challenge in the disaster response phase. Meanwhile, lack of access to adequate mental-health professionals in the early stages of large-scale disasters can dramatically influence the trend of a successful mental health aid. Exploiting the capabilities of mental health professionals can play a great role in the process of effective mental recovery of disaster survivors. Effective coordination of mental health professionals in the complex circumstance of large-scale catastrophes is often crucial, particularly when the number of the health professionals in the disaster-affected area is limited due to absence of a portion of professionals in the scene, the magnitude of the disaster or the high population density of the given area in the early stages of the disaster.

Up to date, in the geospatial industry, the whole attentions have been paid to develop location-based services for smart phones, pocket PCs and tablet devices. Meanwhile, a new generation of communication media, namely tele-operated androids, has been evolving during past few years and to our knowledge less research and application work has been carried out to employ this novel technology in the context of geospatial industry and more specifically for disaster response application domain. Tele-operated android robots are the state of art in the field of humanoid robotics. Their appearance is very humanlike, modelled from a real individual, and so are their motions. A real human can operate them during a remote conversation in order to transfer his presence to the other side of interaction. The new generation of tele-operated android robots is designed to appear and behave like a minimalistic human and therefore allows people from different gender and age to use them in order to transfer and express their presence via this technology. The cellphone-type tele-operated android robots, as a family of this technology and developed based on the same concept, provides a natural and realistic communication between two remote users as if they are facing each other and interacting. In this paper, a conceptual framework has been suggested for adopting cellphone-type tele-operated android robots in the early stages of disasters for providing mental location-based services to individuals. For this purpose, a Peer-to-Peer (P2P) system architecture has been proposed where each cellphonetype tele-operated android robot acts as a peer node coupled with a geo-web processing service which provides the necessary resources as well as process functions that are common for initial responding to the situation and individuals (data collection from the scene, sending collected (geo-) data to center, etc.). The aim of this research study is to add more flexibility and autonomy to GI web services (WMS (de la Beaujardiere, 2006), WFS (Vretanos, 2005), WPS (Schut,

\footnotetext{
* Corresponding author
} 
2009), etc.) and alleviate to some degree the inherent limitations of these centralized systems.

Also, the P2P system architecture takes care of efficient control of connecting each tele-operated android robot to the center(s) where the official or volunteer human mental health professionals are available for controlling the situation remotely. Therefore, the concept of participatory GIS would be used in means of using the benefit of services from official or volunteer trained participators to help the disaster survivors via the proposed web service. However, different modes of connectivity are considered. Requirements are derived, the system architecture is proposed and implementation aspects are discussed. Finally the paper concludes with the challenges that remain to be addressed to make this a reality.

The structure of the paper is as follow. In the next section we introduce the definition and main characteristics of mental health disorders in natural and human made disasters. The research problem is defined and later in section 3 we provide information about cellphone-type tele-operated android robots. Section 4 presents a location based participatory approach for early mental health services during disasters and later the Peerto-Peer system architecture is presented in section 5. Finally, in section 6 the approach, architecture and used technologies are discussed and the paper is finished with concluding remarks and some tips for future research directions.

\section{MENTAL HEALTH DISORDERS IN NATURAL AND HUMAN MADE DISASTERS}

Natural or human made disasters affect the lives of millions of people throughout the world each year. Immediately after the event of a disaster, almost any disaster victim (including disaster survivors and disaster rescue or relief workers) has intrusion or re-experiencing symptoms as he/she finds himself/herself impotent to stop thinking about what happened. Also the high levels of fear, anxiety, efforts to avoid reminders and arousal symptoms may be seen among the disaster victims in the early stages of large-scale disasters (Alexander, 2005; Young, Ford, Ruzek, Friedman, \& Gusman, 1998). The different types of psychosis in the form of stress reactions which exhibit in the disaster survivors after a disaster event can be generally categorized as table 1 (National Center for PTSD, 2010).

\begin{tabular}{|c|l|}
\hline Type & \multicolumn{1}{|c|}{ Characteristics } \\
\hline $\begin{array}{c}\text { Emotional } \\
\text { Reactions }\end{array}$ & $\begin{array}{l}\text { shock, fear, grief, anger, guilt, shame, feeling } \\
\text { helpless, feeling numb, sadness }\end{array}$ \\
\hline $\begin{array}{c}\text { Cognitive } \\
\text { Reactions }\end{array}$ & $\begin{array}{l}\text { confusion, indecisiveness, worry, shortened } \\
\text { attention span, trouble concentrating }\end{array}$ \\
\hline Physical & $\begin{array}{l}\text { tension, fatigue, edginess, insomnia, bodily aches } \\
\text { pain, startling easily, racing heartbeat, nausea, } \\
\text { Reactions } \\
\text { change in appetite }\end{array}$ \\
\hline $\begin{array}{c}\text { Interpersonal } \\
\text { Reactions }\end{array}$ & $\begin{array}{l}\text { distrust, conflict, withdrawal, work or school } \\
\text { problems, irritability, loss of intimacy, feeling } \\
\text { rejected or abandoned }\end{array}$ \\
\hline
\end{tabular}

Table 1. Common reactions in the survivors after the event of disaster

The mental health requirements following a disaster vary according to the disaster impact and exposure and pre-existing vulnerabilities in the community. Some individuals, however, are more likely to have a higher than typical risk for major stress symptoms and Post-traumatic Stress Disorder (PTSD), including those who attributed to the risk factors which are described in table 2 (Alexander, 2005; Bulling \& Abdel
Monem, 2009; National Center for PTSD, 2010; Young, et al., 1998).

\begin{tabular}{|c|l|}
\hline Name & \multicolumn{1}{|c|}{ Characteristics } \\
\hline $\begin{array}{c}\text { Trauma and } \\
\text { Stress }\end{array}$ & $\begin{array}{l}\text { Severe exposure to the disaster, especially injury, } \\
\text { threat to life, and extreme loss. Living in a highly } \\
\text { disrupted or traumatized community. High } \\
\text { secondary stress. }\end{array}$ \\
\hline $\begin{array}{c}\text { Survivor } \\
\text { Characteristics }\end{array}$ & $\begin{array}{l}\text { Female gender, if an adult survivor ages 40 to } \\
\text { 60, being an ethnic minority, low socioeconomic } \\
\text { status, and pre-disaster psychiatric history. }\end{array}$ \\
\hline Family Context & $\begin{array}{l}\text { In an adult survivor, having children n the home. } \\
\text { If female, the presence of a spouse. If a child, the } \\
\text { presence of parental distress. A significantly } \\
\text { distressed family member, interpersonal conflict } \\
\text { or lack of support at home. }\end{array}$ \\
\hline $\begin{array}{c}\text { Resource } \\
\text { Context }\end{array}$ & $\begin{array}{l}\text { Lacking belief in one's ability to cope, few, weak } \\
\text { or deteriorating social resources. }\end{array}$ \\
\hline
\end{tabular}

Table 2. Risk factors for severe stress reactions and lasting PTSD

Apart from the type of stress reactions, the necessity and value of early mental health intervention by the mental health professionals and providing early psychological care for victims of the disasters has been studied and confirmed in several literatures (Everly Jr, 1998).

\section{CELLPHONE-TYPE TELE-OPERATED ANDROID ROBOTS}

Elfoid, a miniature anthropomorphic robot developed at the Advanced Telecommunications Research Institute International (ATR) in Japan (Minato, Nishio, Ogawa, \& Ishiguro, 2012), is an innovative communication medium that conveys individuals' presence to remote locations using voice, appearance, touch, and motion (Figure 1).

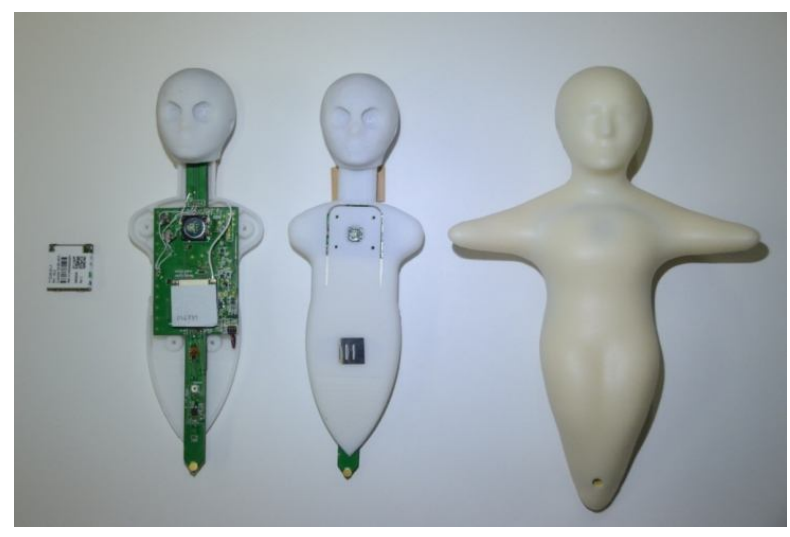

Figure 1. The prototype of cellphone-type android Elfoid

It is a minimal tele-operated android robot combined with cellphone technology that allows people to interact in a way that they can feel each other's presence. The concept of the interface is based on the reproducing user's movement through motioncapture systems plus his or her voice on Elfoid's body, thereby conveying a feeling of user's presence in the same location of the conversation partner (Figure 2). 


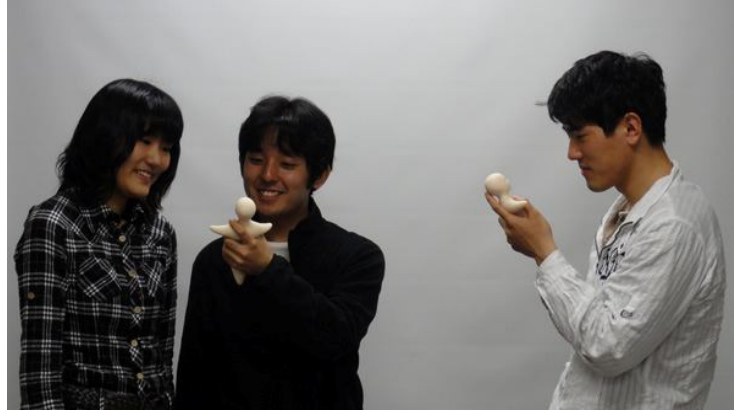

Figure 2. Users hold the Elfoid and make a realistic communication as if they are talking with someone directly

A compact $3 \mathrm{G}$ cellphone unit is embedded in the Elfoid, and users can communicate with others in remote places in the same manner as conventional cellphones. The users of the current prototype of the system can call by pushing a button on the chest of Elfoid. Furthermore, as this device has its own telephone number, it can receive a phone call from others.

Elfoid has a simplified human shape that is designed such that it could be recognized as male or female, old or young, and that users would use their imagination to make the robot more personal. Although the current prototype does not have any sensors and actuators, the future Elfoid is expected to have micro-actuators implemented inside for realization of device's movement. Furthermore, the developed system eventually departs from the button pressing approach currently used in cellular phones, in favor of image and voice recognition technology, with the goal of realizing an intuitive and simple interface for general users (Minato, Sumioka, Nishio, \& Ishiguro, 2012).

Beside the ordinary Elfoid system, the wireless based version of Elfoid also has been developed. This developed system works as a Bluetooth speaker-phone by connecting to a normal cellphone to facilitate the integration of the available smart phones with the Elfoid technology. Hence the users do not need to change their cellphones as their Elfoid system can be connected to the cellphone by the wireless technology.

\section{A LOCATION-BASED PARTICIPATORY APPROACH FOR EARLY MENTAL HEALTH SERVICES DURING DISASTERS}

Lessons learned show that following a disaster, suitable mental health interventions may be neglected or prioritized last in the disaster response phase as providing sufficient number of mental health professionals (particularly in the early stages of the disaster) in the disaster scene and providing the required mental health support services for the disaster survivors is difficult and problematic.

This problem is initiated from several reasons. First of all, supplying the adequate number of mental health professionals from the neighbourhood areas of disaster scene could be a hard and time-consuming task in most cases, especially when the dimensions of the disaster is relatively large. Moreover, the mobility and accessibility to the disaster-affected area are usually encounters with the difficulty for mental health professionals in the early stages of the disaster.

To cope with these usual problems from the aspects of human resource management as well as disaster management, this research suggests a participatory approach for attracting the participation of the mental health professionals which are located away from the disaster-affected area in the decentralized and distributed organization.

To provide a robust and sustainable framework to guarantee the efficient remote distance participation of the mental health professionals, a conceptual model and framework is proposed based on the new technologies in the fields of Geo-informatics. Therefore, the concepts of Geo-Web 2.0 and Location-based Services (LBS) are adopted to develop a geo-spatial processing web service. In order to transfer the presence of the mental health professionals (who are located far from the disasteraffected area) for the disaster survivors (who are located in the disaster-affected area), the geo-spatial location-based technology coupled with humanoid robotics technology by adopting the capabilities of the Elfoid as a cellphone-type teleoperated android robots.

It is believed that employing cellphone-type tele-operated android robots can contribute to a more effective and realistic remote communication between mental health professionals and disaster survivors and facilitate the emotional and mental recovery process. The proposed system has two main categories of the system users: service provider users and service receiver users. The mental health emergency response team is formed from the service provider users and includes mental health professionals (officials and volunteers), the disaster mangers and related staff and authorities.

This category of system users were registered in the proposed location-based service mostly before the disaster strike. The competency and authority of this type of system users has been monitored by the legal authorities and if the competency of personnel was verified and approved, he or she can access to the developed geo-web service for providing the emergency mental health support services to the disaster survivors in the time of disaster event.

The user accessibility level to the service receiver's data (and remote connection to him/her) as a well as different unit of the location-based system by a service provider user is defined by the service administrators based on different parameters. The role of the service provider user in the time of disaster, the professional competency and the specialties of him or her are some of the parameters which determine the accessibility level of a service provider user to the system. The service receiver users include any individual who may encounter with a disaster in the future in the defined supporting operation area of the geo-web service.

The registered service receiver user in this system who has a tele-operated android robot was asked by the system to submit his/her identification information such as national ID number, name, birth date, place of birth, gender, education, income, current address and photograph in the system before the disaster strike. This basic information can be completed with the past physical and mental records beside other related personal information which are provided by the user. However, in the case of availability of a completed and updated national identification database and citizen's health information database, the proposed system may be connected to these data source and the aforementioned information can be retrieved directly from these databases accurately.

To find the location of the cellphone-type tele-operated android robot and associate the spatial dimension to the data which is produced by it in the outdoor application of the device, the device can be equipped by a space-based satellite navigation system such as a GPS unit. However in indoor situations, the location of the device and spatial attribute of the produced data can be acquired by GSM localization techniques or Self- 
reported positioning method. In the proposed system, when a disaster happens, in the first case the service receiver users (disaster survivors) can use tele-operated android robots to connect to the developed geo-web service for the mental health support services (or they could be connected by using help from disaster response team).

On the other hand, in a different mode, the service provider users (professionals) can use the location-based service for searching for tele-operated android robots which are spatially located inside of the disaster-affected area to provide the psychological consultation service. This aim can be reached as the location of any connected tele-operated android robot to the geo-web service is known in the system.

The diagnosis and the treatment process of every service receiver user are recorded in the system by the service provider user in each session of consultation and providing the mental health service and support. The location-based service also can be applied by the service provider or receiver user in allocation and searching practices for the facilities such as the nearest medical centers or field hospitals, if necessary.

As it was mentioned before, the mental health needs and the required support services after a disaster vary by the different parameters such as impact and exposure of the disaster and pre-existing vulnerabilities in the society. Therefore, if the disaster risk and damage maps of the area are available based on the disaster risk and damage assessment analysis, the service receiver users can be prioritized to get the service from the system based on the parameters such as level of the damages in place that the service receiver user located, age, gender and physical and mental records of the individual in the past (level of mental vulnerability).

This spatial decision support unit inside the system for optimized service demand management may play a key role, particularly in early stages of the large-scale disasters, where the number of the required mental health services (demands) is much larger than the available service provider users (supplies).

Since the mental health services in the system were provided in the location-based approach, the further spatio-temporal analysis and spatial data mining practices can be implemented on the system's database to extract the information and transform it into understandable structures for the different stakeholders and authorities in the field of disaster management.

Last but not the least, by employing semantics of geo-data and geo-services (Bakillah, Mostafavi, \& Liang, 2012) as well as quality of geo-data (Mobasheri, 2013), the mental health web processing service can act smarter and more efficient.

\section{SYSTEM ARCHITECTURE}

In this research study, we present a P2P architecture for the location-based service using minimalistic tele-operated android robots by fitting P2P and web services technology into GIS. The proposed framework is based on BestPeer $(\mathrm{Ng}$, Ooi, \& Tan, 2002), and the goal is to develop experimental GIservices in a P2P-based platform. Basically, two types of nodes for services are considered: a large number of tele-operated android robots and PC's (i.e. peers), and a relatively fewer number of Location-Independent Global names Lookup (LIGLO) servers, as shown in Figure 3.

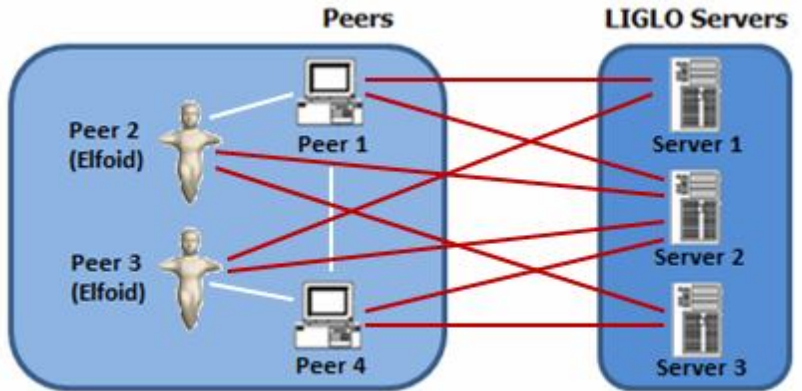

Figure 3. Network topology of peers and servers

The peers could be Elfoid's, GIS services provider, GIS services consumer, as well as UDDI Registries. These services overcome the limitations of existing GI-Services and have the following features (Guan, Wang, \& Zhou, 2004):

- Centralized approaches are generally adopted by current GI-Services, whereas these P2P services adopts a distributed structure built on BestPeer where each peer could take all three roles, i.e., GIS services provider, GIS services consumer and GIS service registrar.

- $\quad$ Each peer in the system can provide GI-services for other peers and may join or depart the P2P services network at any time. This is important when different services need to be offline for update/maintenance reasons.

- The P2P system brings the possibility for services with different development languages, technologies and platforms to communicate with each other.

- $\quad$ The P2P system able to integrate various heterogeneous and autonomous GISs into a cooperative environment which is necessary for disaster response application domain.

The proposed P2P system could be designed and implemented on BestPeer. The services of such a system comprise two kinds of entities, i.e. a large number of normal peer nodes and several LIGLO servers. The former generates a BestPeer Global Identity (BPID) for a peer and maintain peer's current status, and the latter takes the roles of a services provider and a services consumer as well as a services registrar.

Figure 4 illustrates the peer-to-peer architecture and Figure 5 illustrates the internal architecture of a peer node and its connection with other web processing services. Other Web Processing Services may include any kind of services which provide necessary GIS mapping and/or spatio-temporal data processing necessary for disaster response applications. 


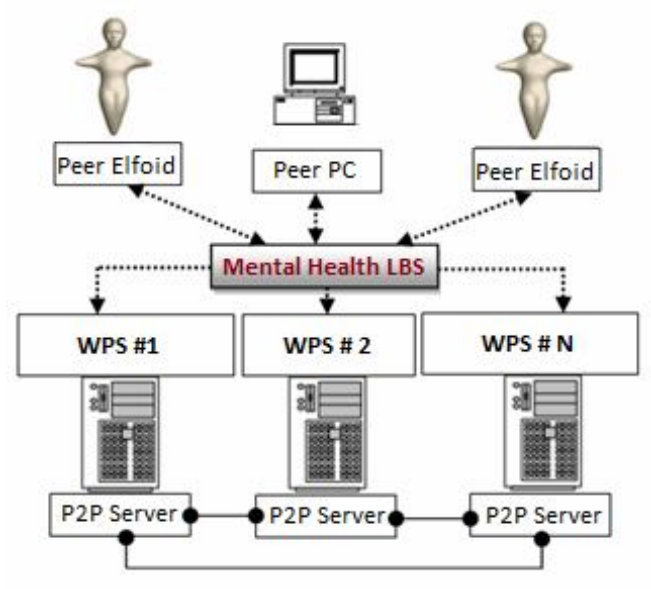

Figure 4. The P2P system Architecture

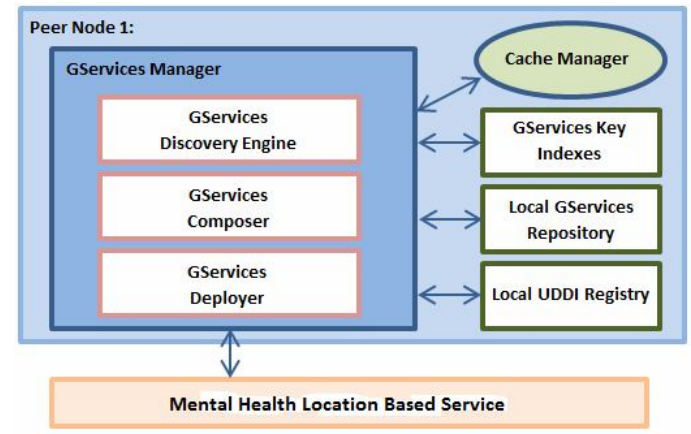

Figure 5. The internal architecture of a peer node and its connection with Mental Health LBS

The system is composed of several components that are loosely integrated. The first component is a Services Manager, which is the most important component of the system. It facilitates geo-data services discovery, services composition and GIservice Deploying. Services Manager consists of three subcomponents, namely the Services Discovery Engine, the Services Composer and the Services Deployer. The publication and location of GI-services are managed by the Discovery Engine. The Composer facilitates the definition of new composite GI-services from existing GI-services, as well as editing existing GI-services (local). The binding and invocation of requested GI-services are handled by the Services Deployer (Guan, et al., 2004).

The second component designed in the system is the GIServices Operating System which provides the environment on which the service operates. Each node in the system has a master agent that manages the GI-services discovery and GIservices description retrieval and would receive GI-services or processed results and send them to other relevant WPSs. The third component is a Cache Manager. This is used in order to cache the results of GI-services discovery and retrieval and reduce the response time of subsequent answers.

Finally, the fourth component is the Web Processing Services, which consists of several modules, corresponding to GIservices discovery and retrieval, GI-services composition and deploying. This service should integrate all the results from different peer nodes, process and pass them in a user-friendly format to the user.

\section{DISCUSSION, CONCLUSION, AND FUTURE WORK}

Providing early mental health services during disaster is a great challenge in the disaster response phase. Lack of access to adequate mental-health professionals in the early stages of large-scale disasters dramatically influences the trend of a successful mental health aid. In this paper we introduced a web processing service for location-based service using minimalistic tele-operated android robots for early mental health interventions in disaster response. This service is supposed to utilize a wide range of technologies, tools and knowledge bases in order to hamper interoperability between various services, servers as well as peer nodes. A peer-to-peer architecture was designed and different components within the architecture were elaborated.

In addition, BestPeer was suggested as the basis for implementing the P2P system. We mentioned that BestPeer introduces a LIGLO server to provide each node with a unique global identity. Therefore, nodes that might have different IP address are known as a single unique entity. LIGLO is a node that has a fixed IP and running LIGLO software, that provides a BestPeer Global Identity for a peer and maintain peer's current status. BestPeer has other advantages that could be useful. BestPeer will always try to make a direct connection to these nodes that have highest priority. In this way, promising peers are first traversed before the less promising ones.

For future work we aim to develop a prototype of mental health LBS based on the proposed architecture and test and evaluate its added value in disaster response application domain. Other possible areas of improvement is to couple this service with other web processing services such as event services or navigation services (Bakillah, Liang, Mobasheri, \& Zipf, 2013) in order to provide more efficient solutions to decision makers and disaster responders.

\section{REFERENCES}

Alexander, D. A. (2005). Early mental health intervention after disasters. Advances in Psychiatric Treatment, 11(1), 12-18.

Bakillah, M., Liang, S. H., Mobasheri, A., \& Zipf, A. (2013). Towards an efficient routing web processing service through capturing real-time road conditions from big data. Paper presented at the Computer Science and Electronic Engineering Conference (CEEC), 20135 th.

Bakillah, M., Mostafavi, M. A., \& Liang, S. H. (2012). Enriching SQWRL queries in support of geospatial data retrieval from multiple and complementary sources Advances in Conceptual Modeling (pp. 241250): Springer.

Bulling, D., \& Abdel Monem, T. (2009). Disaster Mental Health. Wiley Encyclopedia of Forensic Science.

de la Beaujardiere, J. (2006). OpenGIS ${ }^{\circledR}$ web map server implementation specification. Standard Specification, 06-042.

Everly Jr, G. (1998). Emergency mental health: an overview. International journal of emergency mental health, 1(1), 3-7.

Guan, J., Wang, L., \& Zhou, S. (2004). Enabling GIS services in a P2P environment. Paper presented at the Computer and Information Technology, 2004. CIT'04. The Fourth International Conference on. 
Minato, T., Nishio, S., Ogawa, K., \& Ishiguro, H. (2012). Development of cellphone-type tele-operated android. Paper presented at the Proceedings of the 10th Asia Pacific Conference on Computer Human Interaction.

Minato, T., Sumioka, H., Nishio, S., \& Ishiguro, H. (2012). Studying the Influence of Handheld Robotic Media on Social Communications. Social Robotic Telepresence, 15.

Mobasheri, A. (2013). Exploring the Possibility of SemiAutomated Quality Evaluation of Spatial Datasets in Spatial Data Infrastructure. Journal of ICT Research \& Applications, 7(1).

National Center for PTSD. (2010). Mental Health Reactions After Disaster: U.S. Department of Veterans Affairs.

Ng, W. S., Ooi, B. C., \& Tan, K.-L. (2002). Bestpeer: A selfconfigurable peer-to-peer system. Paper presented at the 8th International Conference on Data Engineering.

Schut, P. (2009). Corrigendum for OpenGIS Implementation standard Web Processing Service (WPS) 1.0.0: Open Geospatial Consortium Specification.

Vretanos, P. A. (2005). Web feature service implementation specification. Open Geospatial Consortium Specification, 04-094.

Young, B. H., Ford, J. D., Ruzek, J. I., Friedman, M. J., \& Gusman, F. D. (1998). Disaster mental health services: A guidebook for clinicians and administrators: National Center for Post-Traumatic Stress Disorder. 